\title{
Comparación del ruido producido en el túnel San Eduardo y cerro Santa Ana, ubicados en la ciudad de Guayaquil
}

\section{Comparison of the noise produced in the san Eduardo tunnel and Santa Ana hill, located in the city of Guayaquil}

Ing. Marco Vinicio Noroña Merchán

Universidad Internacional del Ecuador, Ecuador

Autor para correspondencia: manoroname@uide.edu.ec

Fecha de recepción: 15 de Septiembre 2017 - Fecha de aceptación: 15 de Enero de 2018

Resumen: En este artículo científico se realizara la comparación de ruido que se produce en horas donde hay gran cantidad de tráfico como hora de almuerzo (12-1:30 pm) y hora de salida del trabajo (5-6:30 pm) en los túneles San Eduardo y Cerro Santa Ana, ubicados en la ciudad de Guayaquil-Ecuador, esto con la ayuda de una aplicación móvil (Decibel Meter) previamente descargada en teléfonos celulares con tecnología IOS (IPhone 5 s) y Android (Sony Xperia Z2) respectivamente; así poder conocer la variación de valores obtenidos, y el porqué de la variación de medidas, para determinar en cuál de los túneles antes mencionados se produce una mayor cantidad de ruido y saber si está dentro de los parámetros aceptados en cuanto a la contaminación sonora ; así mismo el daño que produciría si se expone por un periodo determinado al ruido que se genera o el tiempo permitido que un ser humano podría estar expuesto sin que sea perjudicial para su salud, previo al umbral de dolor.

Palabras Clave: contaminación sonora; ruido; umbral de dolor

\begin{abstract}
In this scientific article, the comparison of noise that occurs in hours where there is a large amount of traffic such as lunch time (12-1: $30 \mathrm{pm}$ ) and departure time from work (5-6: 30 pm) in the tunnels San Eduardo and Cerro Santa Ana, located in the city of Guayaquil-Ecuador, this with the help of a application mobile (APP) previously downloaded in cell phones with IOS technology (Iphone $5 \mathrm{~s}$ ) and Android (Sony Xperia Z2) respectively; so to be able to know the variation of values obtained, and the reason for the variation of measurements, to determine in which of the aforementioned tunnels there is a greater amount of noise and to know if it is within the accepted parameters in terms of noise pollution; Likewise the damage that would occur if exposed for a specific period to the noise that is generated or the time allowed that a human being could be exposed without being harmful to their health, prior to the pain threshold.
\end{abstract}

Key words: noise; pain threshold; pollution 


\section{Introducción}

En base a una investigación con la implementación de las tecnologías más utilizadas en la actualidad; como lo son los Smartphone, se comprueba la diferencia del número de decibeles en dos de los túneles de la ciudad de Guayaquil en horas transitadas; en este caso las medidas fueron tomadas entre las 12:30 PM - 1:30 PM, tomando en cuenta los límites sonoros según la normativa del Ministerio del Medio Ambiente y el estudio de la afectación sonora en una persona.

En los túneles antes mencionados existe una gran cantidad de ruido debido al cerramiento en los mismos, es el motivo por el que al momento de existir ruido este retumba entre las paredes sin tener como opción a un desfogue causando menos inconformidad sonora.

\section{Materiales y Métodos}

En este trabajo usamos las limitaciones establecidas según el Ministerio del Medio Ambiente, los cuales fueron medidos mediante una aplicación en dos Smartphone de diferentes marcas, el primero fue un Sony Xperia z2 con sistema operativo Android (Decibeles Meter) y un iPhone 5 con sistema operativo IOS (Decibel X) una vez descargadas y calibradas las aplicaciones nos dirigimos a cada uno de los túneles en un vehículo para proceder a realizar el estudio antes mencionado.

Sonómetro -. Es un instrumento de medición de ruidos o niveles al medir la presión del sonido. Su unidad de medida acústica es el decibel (dB) (Jiménez Padilla, 2012).

Decibel (dB)-. Unidad adimensional utilizada para expresar el logaritmo de la razón entre una cantidad medida y una cantidad de referencia. El decibel es utilizado para describir niveles de presión, de potencia o de intensidad sonora (Jiménez Padilla, 2012).

\section{Ruidos producidos por vehículos automotores}

La Entidad Ambiental de Control establecerá, en conjunto con la autoridad policial competente, los procedimientos necesarios para el control y verificación de los niveles de ruido producidos por vehículos automotores (Ministerio de Transporte y Obras Públicas, 2013).

De la medición de niveles de ruido producidos por vehículos automotores. - las mediciones destinadas a verificar los niveles de presión sonora arriba indicados, se efectuarán con el vehículo estacionado, a su temperatura normal de funcionamiento, y acelerado a $3 / 4$ de su capacidad. En la medición se utilizará un instrumento decibelímetro, normalizado, previamente calibrado, con filtro de ponderación A y en respuesta lenta.

El micrófono se ubicará a una distancia de 0,5 m del tubo de escape del vehículo siendo ensayado, y a una altura correspondiente a la salida del tubo de escape, pero que en ningún caso será inferior a $0,2 \mathrm{~m}$. El micrófono será colocado de manera tal que forme un ángulo de 45 grados con el plano vertical que contiene la salida de los gases de escape. En el caso de vehículos con descarga vertical de gases de escape, el micrófono se situará a la altura del orificio 
de escape, orientado hacia lo alto y manteniendo su eje vertical, y a 0,5 $\mathrm{m}$ de la pared más cercana del vehículo.

Tabla 1. Niveles de Presión Sonora Máxima Para Vehículos

\begin{tabular}{|c|c|c|}
\hline $\begin{array}{l}\text { Categoría } \\
\text { De Vehic ulo }\end{array}$ & Descripción & $\begin{array}{l}\text { Nps } \\
\text { Máx. } \\
\text { (D ba) }\end{array}$ \\
\hline \multirow[t]{3}{*}{ Motocicletas: } & De hasta 200 centímetros cúbicos. & 80 \\
\hline & Entre 200 y 500 c. c. & 85 \\
\hline & Mayores a $500 \mathrm{c}$ c. & 86 \\
\hline \multirow[t]{4}{*}{ Vehículos: } & $\begin{array}{l}\text { Transporte de personas, conductor, } 9 \\
\text { asiento. }\end{array}$ & 80 \\
\hline & $\begin{array}{l}\text { Transp orte de personas, conductor, } 9 \\
\text { asientos, y peso menor a } 3,5 \text { toneladas. }\end{array}$ & 81 \\
\hline & $\begin{array}{l}\text { Transporte de personas, conductor, } 9 \\
\text { asientos, y peso mayor a } 3,5 \text { toneladas. }\end{array}$ & 82 \\
\hline & $\begin{array}{l}\text { Transporte de personas, conductor, } 9 \\
\text { asientos, peso mayor a } 3,5 \mathrm{~T} \text {, potencia } \\
\text { mayor a } 200 \mathrm{HP} \text {. }\end{array}$ & 85 \\
\hline \multirow{3}{*}{$\begin{array}{l}\text { Vehículos de } \\
\text { Carga: }\end{array}$} & Peso máximo hasta $3,5 \mathrm{~T}$ & 81 \\
\hline & Peso máximo de $3,5 T$ hasta 12,0 & 86 \\
\hline & Peso máximo mayor a $12,0 \mathrm{~T}$ & 88 \\
\hline
\end{tabular}

Fuente: (Municipio del Distrito Metropolitano de Quito, 2015)

\section{Resultados y Discusión}

Para la medición de decibeles en los dos túneles de la ciudad de Guayaquil pasamos por medio de estos y se utilizó una aplicación llamada decibels meters, la cual después de una prueba resultó ser la más acertada en el rango de medidas, la diferencia entre ambos túneles es que variaron los decibeles por la cantidad de vehículos en los mismos; en las imágenes se pueden observar que en la figura 1 en el túnel del Cerro Santa Ana la medida más baja fue de $48 \mathrm{~dB}$, mientras que la más alta fue de $58 \mathrm{~dB}$, en el caso de del túnel San Eduardo la variación fue mínima; ya que, existía menos tránsito de vehículo; por lo cual la medida más baja fue de $47 \mathrm{~dB}$ y la más alta alcanzó los 56 dB. 


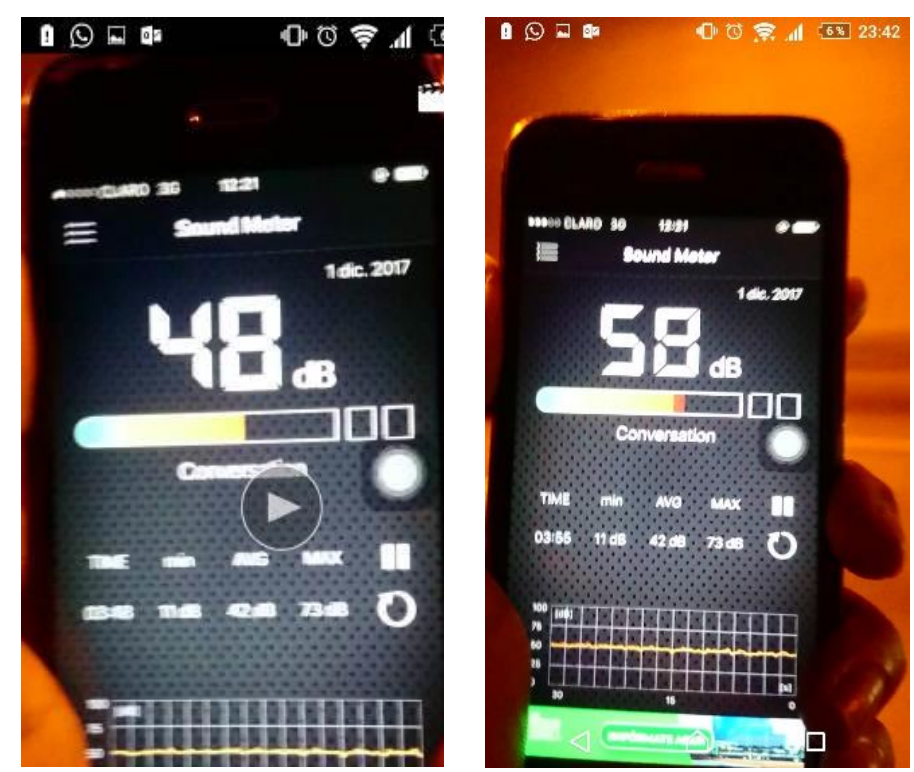

Figura 1. Variación de Medidas, Túnel Cerro Santa Ana
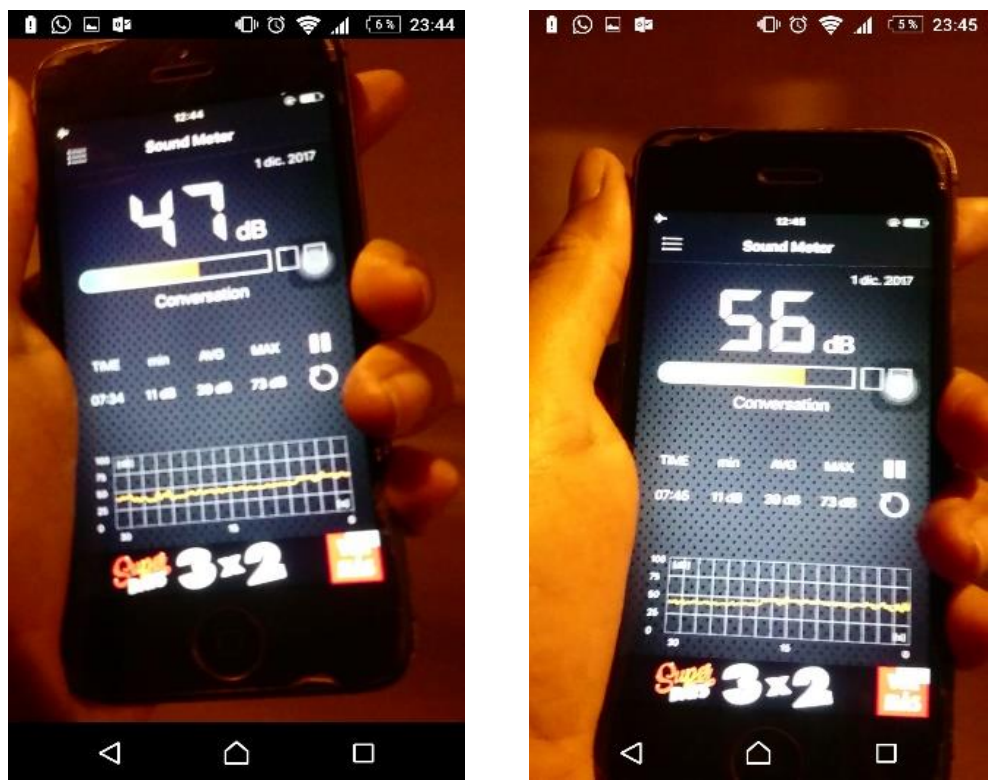

Figura 2. Variación de Medidas, Túnel San Eduardo Fuente: El Autor

\section{Conclusiones}

Según los datos obtenidos de los dos túneles se puede decir que como conclusión a la investigación previamente realizada se obtuvieron ciertos resultados; donde se puede apreciar que en el túnel del Cerro Santa Ana transitan mayor cantidad de vehículo; por lo que, la cantidad de decibeles era mayor, en comparación a la del túnel San Eduardo donde el número de decibeles disminuyó por la poca transición de vehículos. 
Después de la toma de medidas y comparación de ruidos producidos en los túneles, se pudo notar que, a mayor longitud de los mismos, existirá una mayor cantidad de contaminación ambiental, la cual es directamente proporcional con el tráfico vehicular en horas pico.

\section{Bibliografía}

Bartés, A. P. (2005). Métodos estadísticos. Control y mejora de la calidad. Catalunya: Universitat Politècnica de Catalunya.

Bosch, R. (2012). Gestión del motor de gasolina. Reverté S.A.

Bosch, R. (sf). Manual de la técnica del automóvil, 4ta Edición. Reverté S.A.

Callejo, D. G. (2014). Mantenimiento mecánico preventivo del vehículo. España: Paraninfo.

Company, F. M. (09 de Enero de 2015). Ford Service Manual. North América.

Jilliane, E. (septiembre de 2016). Cómo Calcular el Consumo de Gasolina.

Jiménez Padilla, B. (2012). Técnicas básicas de mecánica de vehículos. Malaga: IC Editorial.

Julián Ferrer, Gema Checa. (s.f.). Mantenimiento Mecánico Preventivo del vehículo. Editex.

Ministerio de Transporte y Obras Públicas. (2013). Procedimientos de Operación y Seguridad Vial. Recuperado el 2017 de Diciembre de 2017, de https://cauchosvikingo.com/wpcontent/uploads/2017/07/Manual_NEVI-12_VOLUMEN_5-Procedimiento-de-operaciony-seguridad-vial.pdf

Municipio del Distrito Metropolitano de Quito. (2015). Normativas. Recuperado el 1 de Diciembre de 2017, de http://www.ecuadorambiental.com/doc/normas_tecnicas.pdf

Normalización, I. E. (s.f.). Normas INEN. Obtenido de https://law.resource.org/pub/ec/ibr/ec.nte.2203.2000.pdf

PCE. (s.f.). Sound Level Meter. Recuperado el 1 de Diciembre de 2017, de https://www.pceinstruments.com/us/measuring-instruments/test-meters/sound-level-meter-noise-lev

Pardiñas, J. (2013). Sistemas Auxiliares del Motor. Editex.

Puerto Martín A, J.A García Rodríguez. (s.f). La Contaminación Atmosférica. Salamanca. 